$05,11,10$

\title{
Влияние магнитного поля на термодинамические и магнитные свойства антиферромагнитной модели Изинга на объемно-центрированной кубической решетке
}

\author{
(C) А.К. Муртазаев ${ }^{1}$, М.К. Рамазанов ${ }^{1}$, К.Ш. Муртазаев ${ }^{1,2}$, М.А. Магомедов ${ }^{1,2}$, М.К. Бадиев ${ }^{1}$ \\ ${ }^{1}$ Институт физики ДагНЦ РАН, \\ Махачкала, Россия \\ 2 Дагестанский государственный университет, \\ Махачкала, Россия \\ E-mail: 5kurban@mail.ru
}

Поступила в Редакцию 9 июля 2019 г.

В окончательной редакции 1 октября 2019 г.

Принята к публикации 2 октября 2019 г.

Репличным алгоритмом метода Монте-Карло изучено влияние внешнего магнитного поля на фазовые переходы, термодинамические и магнитные свойства трехмерной антиферромагнитной модели Изинга на объемно-центрированной кубический решетке с учетом взаимодействий вторых ближайших соседей. Построена фазовая диаграмма зависимости критической температуры от величины внешнего магнитного поля. Показано, что в рассмотренном интервале значений магнитного поля наблюдается фазовый переход второго рода.

Ключевые слова: модель Изинга, метод Монте-Карло, фазовые перехоы, магнитное поле, спиновая система.

DOI: $10.21883 /$ FTT.2020.02.48872.553

\section{1. Введение}

В настоящее время в физике конденсированного состояния повышенный интерес представляют исследования магнитных и термодинамических свойств фрустрированных спиновых систем. Фрустрированные спиновые системы обладают необычными магнитными свойствами, имеют богатое разнообразие фаз и фазовых переходов (ФП), обусловленные сильным вырождением и высокой чувствительностью фрустрированных систем к различным внешним факторам, в том числе и магнитным полям. Влияние внешнего магнитного поля и учет взаимодействий первых и вторых ближайших соседей может играть важную роль в поведении таких систем [1-6].

В данной работе нами изучается влияние магнитного поля на характер ФП, магнитные и термодинамические свойства антиферромагнитной модели Изинга на объемно-центрированной кубической (ОЦК) решетке с учетом взаимодействий первых и вторых ближайших соседей.

Теоретические и численные исследования модели Изинга на разных типах решеток и с конкуренцией обменных взаимодействий были проведены в работах $[6-11]$.

В работе [6] нами методом Монте-Карло (МК) для модели Изинга на ОЦК решетке были получены все магнитные структуры основного состояния и построена фазовая диаграмма. В этой же работе были обнаружены области на фазовой диаграмме, где наблюдались ФП первого и второго рода. Это подтверждает влияние дополнительных факторов, таких как взаимодействие вторых ближайших соседей, на природу ФП данной модели. Теоретические исследования, проведенные в работе [7] согласуются с данными, полученными в работе [6] и показывают, что для модели Изинга на ОЦК-решетке имеет место ФП второго рода. В работе [10] для данной модели рассчитаны значения всех основных статических критических индексов и установлены особенности критического поведения в зависимости от величины взаимодействия вторых ближайших соседей. Авторы работы [12] исследовали квантовую антиферромагнитную модель Изинга с произвольным значением спина и с учетом внешнего магнитного поля. В этой работе получены общие аналитические выражения для фрустрационного поля и показано, что внешнее магнитное поле влияет на физические свойства данной модели.

Интерес к антиферромагнитной модели Изинга на ОЦК-решетке обусловлен еще и тем, что влияние внешнего магнитного поля на ФП, магнитные и термодинамические свойства этой модели на основе современных методов до сих пор никем не проводилось. При изучении таких систем до сих пор основное внимание уделялось моделям на квадратной, треугольной и гексагональной решетке [13-21].

Исследование этой модели на основе современных методов и идей позволит получить ответ на ряд вопросов, связанных с ФП, магнитными и термодинамическими свойствами спиновых систем, а также влияния на них внешнего магнитного поля. 


\section{2. Модель и метод исследования}

Гамильтониан антиферромагнитной модели Изинга на ОЦК-решетке с учетом взаимодействия первых и вторых ближайших соседей, а также внешнего магнитного поля имеет следующий вид:

$$
H=-J_{1} \sum_{\langle i, j\rangle}\left(S_{i} S_{j}\right)-J_{2} \sum_{\langle i, j\rangle}\left(S_{i} S_{j}\right)-H \sum_{\langle i\rangle} S_{l},
$$

где $J_{1}$ и $J_{2}$ - константы обменного антиферромагнитного взаимодействия первых $\left(J_{1}<0\right)$ и вторых $\left(J_{2}<0\right)$ ближайших соседей, $S_{i, j, l}= \pm 1-$ изинговский спин, $H$ - величина магнитного поля (величина магнитного поля приводится в единицах $\left.H /\left|J_{1}\right|\right)$. В данной работе рассматривается случай, когда $r=\left|J_{2}\right| /\left|J_{1}\right|=1.0$.

Классический алгоритм метода МК сталкивается с рядом проблем, связанных с выводом системы в термодинамическое равновесие в области критических температур. Это обстоятельство еще сильнее усугубляется, когда речь идет о системах с конкурирующими обменными взаимодействиями и фрустрациями. Использование специально разработанных репличных алгоритмов метода МК позволяет с большой точностью исследовать такие системы $[5,7,8,10]$. Поэтому, в данной работе нами использовался высокоэффективный репличный алгоритм метода МК.

Расчеты проводились для систем с периодическими граничными условиями (ПГУ) и линейными размерами $2 \times L \times L \times L=N, L=18-90$, где $L$ измеряется в размерах элементарной ячейки. Для анализа природы и характера ФП использовались гистограммный метод и метод кумулянтов Биндера. Величина значений внешнего магнитного поля менялось в интервале $0.0 \leq H \leq 6.0$. Для вывода системы в состояние термодинамического равновесия отсекался участок длиной $\tau_{0}=4 \cdot 10^{5}$ шагов МК на спин, что в несколько раз больше длины неравновесного участка. Усреднение термодинамических параметров проводилось вдоль марковской цепи длиной до $\tau=500 \tau_{0}$ шагов МК на спин.

\section{3. Результаты моделирования}

Для построения температурных зависимостей восприимчивости $\chi$ и теплоемкости $C$ использовались выражения

$$
\begin{gathered}
\chi= \begin{cases}(N K)\left(\left\langle m^{2}\right\rangle-\langle m\rangle^{2}\right), & T<T_{N}, \\
(N K)\left\langle m^{2}\right\rangle, & T \geq T_{N},\end{cases} \\
C=\left(N K^{2}\right)\left(\left\langle U^{2}\right\rangle-\langle U\rangle^{2}\right),
\end{gathered}
$$

где $N$ - число частиц, $K=\left|J_{1}\right| / k_{B} T, U-$ внутренняя энергия, $m$ - параметр порядка, $T_{N}-$ критическая температура ( $U$ и $m$ являются нормированными величинами).

Параметр порядка системы определялся по формуле

$$
m=3 m_{1}-m_{2}-m_{3}-m_{4},
$$

где $m_{1}, m_{2}, m_{3}, m_{4}-$ параметры порядка по подрешеткам.

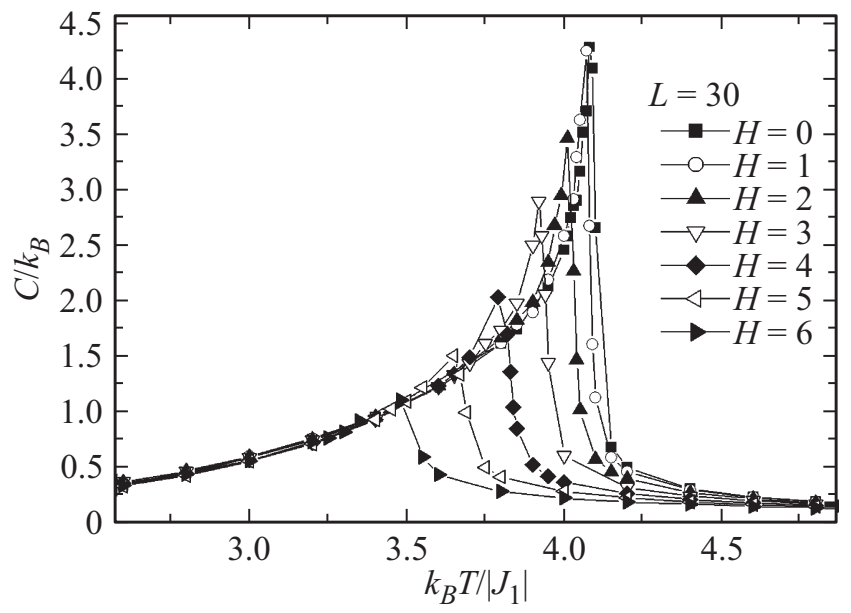

Рис. 1. Зависимость теплоемкости $C / k_{B}$ от температуры $k_{B} T /\left|J_{1}\right|$ для разных значений $H$.

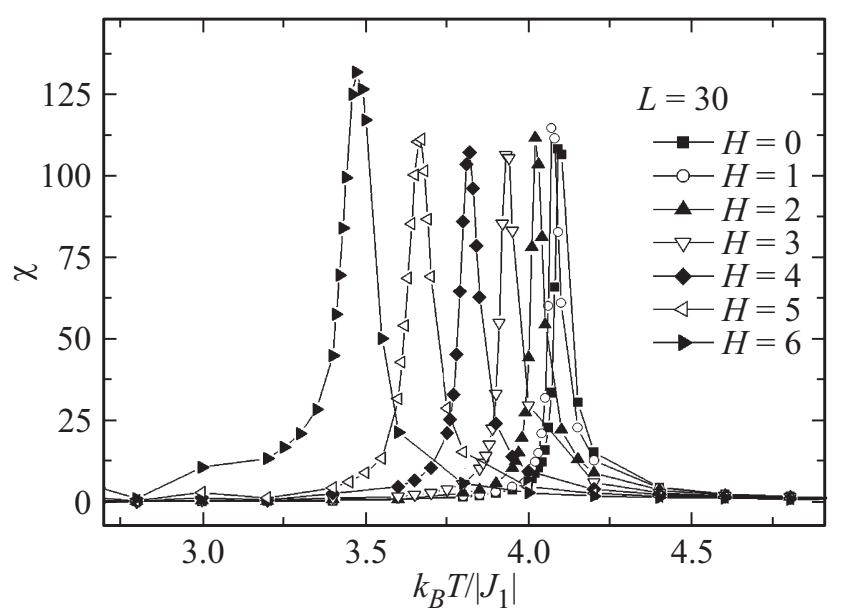

Рис. 2. Зависимость восприимчивости $\chi$ от температуры $k_{B} T /\left|J_{1}\right|$ для разных значений $H$.

Намагниченность вычислялась по формуле

$$
M=\frac{1}{N} \sum_{(i)} S_{i}
$$

Для определения рода ФП и нахождения критический температуры $T_{N}$ был использован метод кумулянтов Биндера $U_{L}$ четвертого порядка

$$
U_{L}=1-\frac{\left\langle m^{4}\right\rangle_{L}}{3\left\langle m^{2}\right\rangle_{L}^{2}}
$$

C помощью выражения (6) с большой точностью можно определить критическую температуру $T_{N}$ для ФП второго рода [10].

На рис. 1 и 2 приведены температурные зависимости теплоемкости и восприимчивости при $L=30$ для различных значений магнитного поля.

На этих рисунках видно, что с увеличением значений $H$ наблюдается сдвиг максимумов теплоемкости и 
восприимчивости в сторону более низких температур. Одновременно наблюдается спад абсолютных значений максимумов теплоемкости. Сдвиг максимумов теплоемкости и восприимчивости в сторону более низких температур обусловлен тем, что магнитное поле усиливает конкурирующее взаимодействие вторых ближайших соседей. Спад абсолютных значений максимумов теплоемкости происходит за счет уменьшения флуктуаций.

На рис. 3 приведены характерные зависимости магнитного параметра порядка от температуры для различных значений $H$. На рисунке видно, что с увеличением $H$ спад магнитного параметра порядка смещается в сторону более низких температур. Вместе с этим уменьшается значение магнитного параметра порядка в области низких температур. Это объясняется усилением конкурирующего взаимодействия вторых ближайших соседей с ростом магнитного поля.

Зависимости намагниченности от температуры для различных значений $H$ приведены на рис. 4. Для ну-

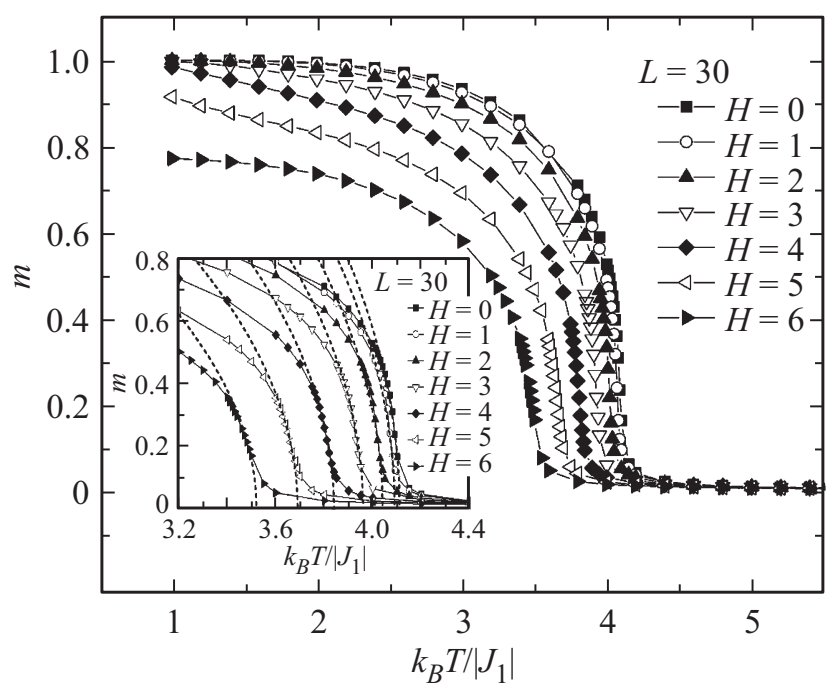

Рис. 3. Зависимость параметра порядка $m$ от температуры $k_{B} T /\left|J_{1}\right|$ для разных значений $H$.

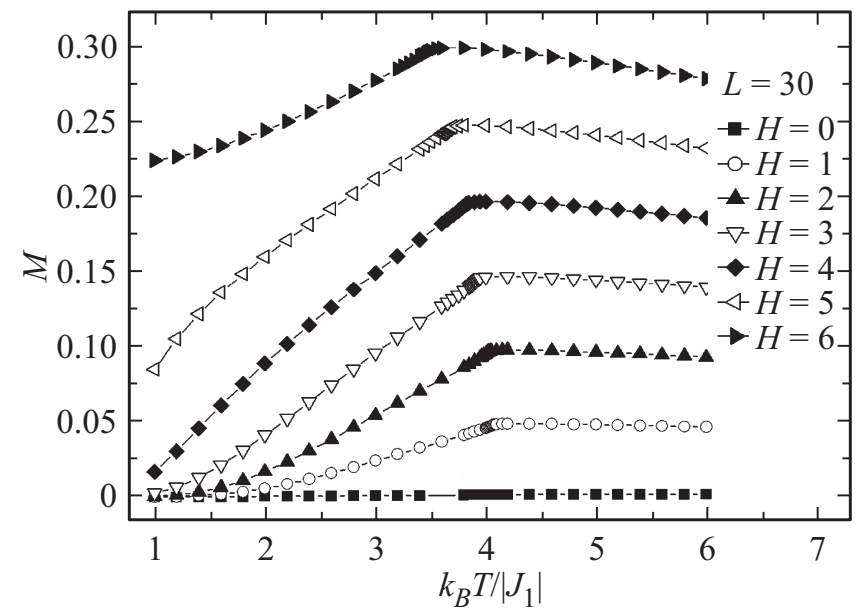

Рис. 4. Зависимость намагниченности $M$ от температуры $k_{B} T /\left|J_{1}\right|$ для разных значений $H$.

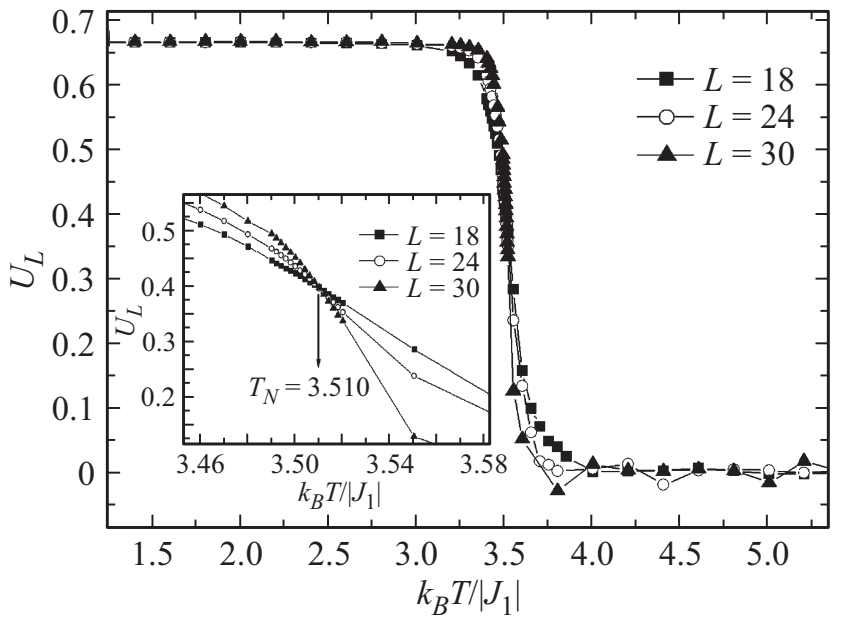

Рис. 5. Зависимость кумулянта Биндера $U_{L}$ от температуры $k_{B} T /\left|J_{1}\right|$ для разных $L$ при $H=6$.

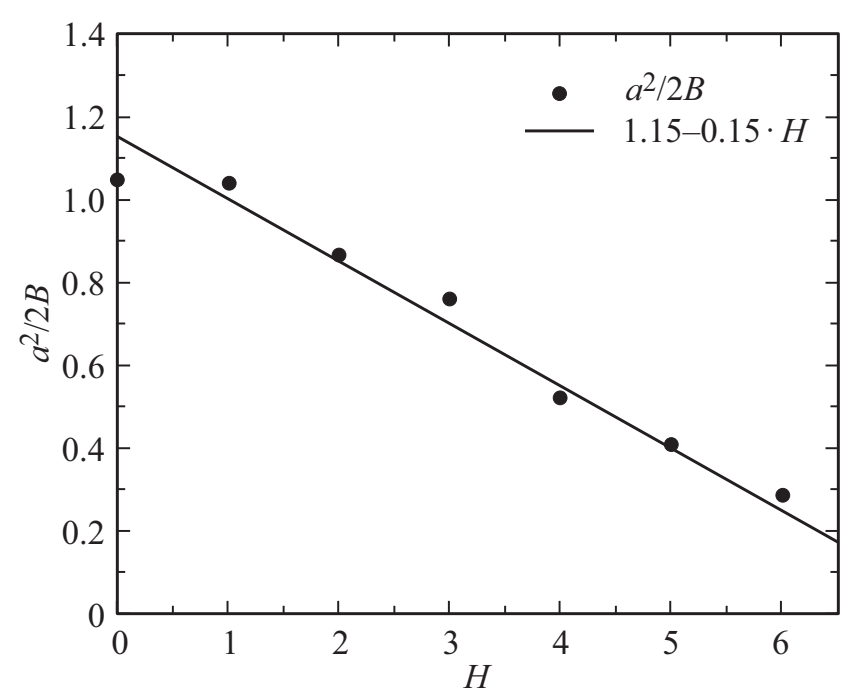

Рис. 6. Зависимость коэффициента $\frac{a^{2}}{2 B}$ от значения магнитного поля $H$.

левого значения магнитного поля, как и ожидалось намагниченность равна нулю. С увеличением $H$ растет намагниченность системы. На рисунке видно, что намагниченность до температуры ФП растет, после чего наблюдается спад из-за тепловых флуктуаций. Чем больше значение магнитного поля, тем сильнее выражается спад после температуры ФП.

Зависимости кумулянта Биндера $U_{L}$ от температуры при $H=6$ для разных значений $L$ представлены на рис. 5. На рисунке видна четко выраженная точка пересечения $\left(T_{N}=3.510\right)$, которая является критический температурой и свидетельствует о ФП второго рода.

Для анализа результатов, полученных в данной работе в рамках теории Ландау, использовалось следующее выражение [22]

$$
C=C_{0}+\frac{a^{2}}{2 B} T_{N}
$$


Значения критических температур, разности теплоемкостей и коэффициента $\frac{a^{2}}{2 B}$ при различных значениях магнитного поля

\begin{tabular}{c|c|c|c|c|c|c|c}
\hline$H$ & 0 & 1 & 2 & 3 & 4 & 5 & 6 \\
\hline$T_{N}$ & 4.11 & 4.09 & 4.04 & 3.96 & 3.84 & 3.69 & 3.51 \\
$C-C_{0}$ & 4.3 & 4.25 & 3.5 & 3 & 2.0 & 1.5 & 1 \\
$\frac{a^{2}}{2 B}$ & 1.046 & 1.039 & 0.866 & 0.758 & 0.521 & 0.407 & 0.284
\end{tabular}

Из этого выражения следует, что

$$
\frac{a^{2}}{2 B}=\frac{C-C_{0}}{T_{N}}
$$

где $C$ - теплоемкость несимметричной фазы, $C_{0}-$ теплоемкость симметричной фазы.

Значения критических температур, разности теплоемкостей и коэффициента $\frac{a^{2}}{2 B}$, рассчитанного с помощью выражения (8), для различных значений магнитного поля приведены в таблице.

На рис. 6 приведена зависимость коэффициента $\frac{a^{2}}{2 B}$ от значения магнитного поля $H$. Как видно из рисунка все точки в пределах погрешности хорошо ложатся на прямую, что позволяет говорить о линейной зависимости $\frac{a^{2}}{2 B}$ от $H$.

На вставке рис. 3 приведены пунктирные кривые, описывающие параметр порядка, полученные из теории Ландау с помощью выражения

$$
m=\sqrt{\frac{a}{2 B}\left(T_{N}-T\right)} .
$$

На рисунке видно, что вблизи критической области наблюдается хорошее соответствие данных, полученных как численным методом, так и построенных в рамках теории фазового перехода второго рода.

Для более подробного анализа природы ФП нами был использован гистограммный метод анализа данных.

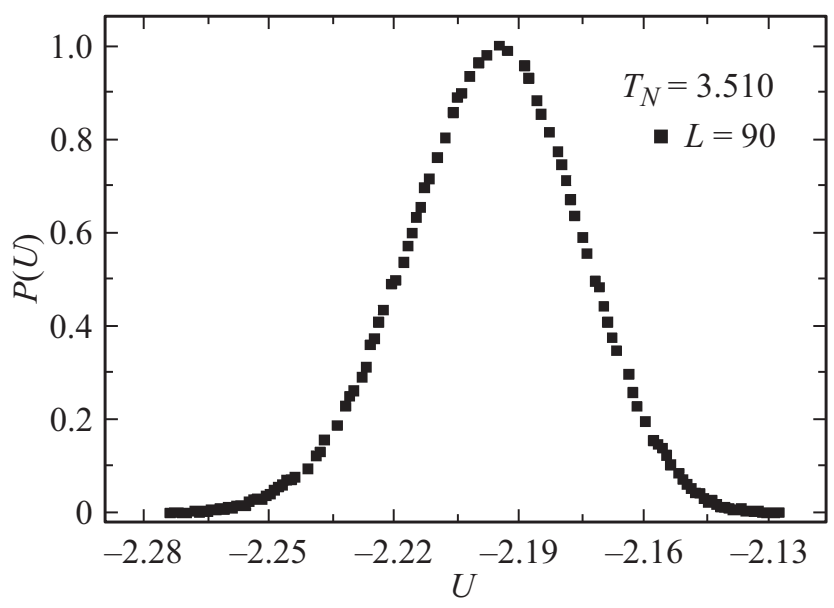

Рис. 7. Гистограмма распределения энергии для $H=6.0$.

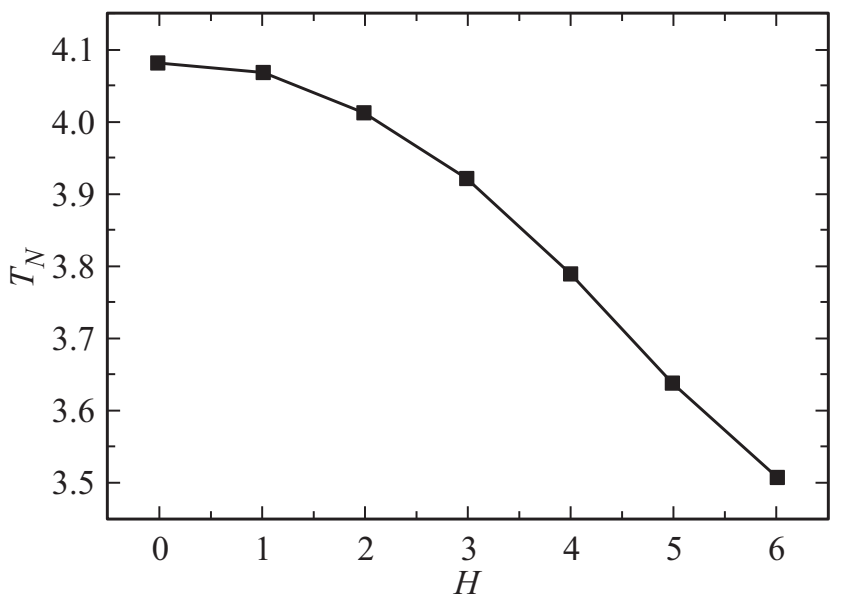

Рис. 8. Фазовая диаграмма зависимости критической температуры $T_{N}$ от величины магнитного поля.

На рис. 7 видно, что гистограмма распределения энергии для систем с линейным размером $L=90$ при $H=6.0$ имеет один пик. Это говорит о том, что в системе реализуется ФП второго рода. График построен при критической температуре $T_{N}=3.510$. Аналогичные гистограммы были построены для всех значений поля в интервале $0.0 \leq H \leq 6.0$. Наши данные свидетельствуют о том, что в рассмотренном интервале значений $H$ реализуется ФП второго рода.

На рис. 8 представлена фазовая диаграмма зависимости критической температуры $T_{N}$ от величины магнитного поля $H$. Показано, что с увеличением величины магнитного поля в интервале $0.0 \leq H \leq 6.0$ температура ФП уменьшается. В рассмотренном интервале значений магнитного поля переход из антиферромагнитной фаза в парамагнитную осуществляется как ФП второго рода.

\section{4. Заключение}

Выполнено исследование трехмерной антиферромагнитной модели Изинга на объемно-центрированной кубической решетке с учетом взаимодействия первых и вторых ближайших соседей, а также внешнего магнитного поля. Рассмотрен интервал значений величины магнитного поля $0.0 \leq H \leq 6.0$. Установлено, что для всех значений магнитных полей в рассмотренном интервале наблюдается фазовый переход второго рода.

\section{Финансирование работы}

Исследование выполнено при финансовой поддержке Российского фонда фундаментальных исследований в рамках научных проектов № 18-32-00391-мол-а, № 1902-00153-a, № 18-32-20098-мол-а-вед.

\section{Конфликт интересов}

Авторы заявляют, что у них нет конфликта интересов. 


\section{Список литературы}

[1] А.З. Паташинский, В.Л. Покровский. Флуктуационная теория фазовых переходов. Наука, М. (1982).

[2] Ш. Ма. Современная теория критических явлений. Мир, M. (1980).

[3] Р.С. Гехт. УФН 159, 261 (1989).

[4] K. Katsumata, H. Aruga Katori, S. Kimura, Y. Narumi, M. Hagiwara, K. Kindo. Phys. Rev. B 82, 104402 (2010).

[5] А.К. Муртазаев, М.К. Рамазанов, Д.Р. Курбанова, М.К. Бадиев. ФТТ 60, 1162 (2018).

[6] A.K. Murtazaev, M.K. Ramazanov, D.R. Kurbanova, M.A. Magomedov, K.Sh. Murtazaev. Mater. Lett. 236, 669 (2019).

[7] А.К. Муртазаев, М.К. Рамазанов, Ф.А. Кассан-Оглы, Д.Р Курбанова. ЖЭТФ 147, 127 (2015).

[8] A.K. Murtazaev, M.K. Ramazanov, M.K. Badiev. Physica B 476, 1 (2015).

[9] M.K. Ramazanov, A.K. Murtazaev, M.A. Magomedov. Physica A 521, 543 (2019).

[10] А.К. Муртазаев, М.К. Рамазанов, Д.Р. Курбанова, М.К. Бадиев, Я.К. Абуев. ФТТ 59, 1082 (2017).

[11] Ф.А. Кассан-Оглы, А.И. Прошкин. ФТТ 60, 6 (2018).

[12] A.I. Proshkin, F.A. Kassan-Ogly. Mater. Sci. Forum 845, 93 (2016).

[13] H. Kawamura. J. Phys. Soc. Jpn. 61, 1299 (1992).

[14] A. Mailhot, M.L. Plumer, A. Caille. Phys. Rev. B 50, 6854 (1994).

[15] Л.Е. Свистов, А.И. Смирнов, Л.А. Прозорова, О.А. Петренко, А.Я. Шапиро, Л.Н. Демьянц. Письма в ЖЭТФ 80, 231 (2004).

[16] А.К. Муртазаев, М.А. Магомедов, М.К. Рамазанов. Письма в ЖЭТФ 107, 265 (2018).

[17] М.К. Рамазанов, А.К. Муртазаев. Письма в ЖЭТФ 103, 522 (2016).

[18] М.К. Рамазанов, А.К. Муртазаев. Письма в ЖЭТФ 106, 72 (2017).

[19] A.K. Murtazaev, M.K. Ramazanov, M.K. Badiev. Physica A 507, 210 (2018).

[20] F.A. Kassan-Ogly, A.K. Murtazaev, A.K. Zhuravlev, M.K. Ramazanov, A.I. Proshkin. J. Magn. Magn. Mater. 384, 247 (2015).

[21] M.K. Ramazanov, A.K. Murtazaev, M.A. Magomedov. Solid State Commun. 233, 35 (2016).

[22] Л.Д. Ландау, Е.М. Лифшиц. Теоретическая физика. Т. V. Ч. 1. Наука, М. (1976).

Редактор Т.Н. Василевская 